\title{
AVALIAÇÃO DO CONHECIMENTO DE MÉDICOS E EQUIPE DE ENFERMAGEM NAS OCORRÊNCIAS DE SEPSE.
}

\author{
MEDICAL KNOWLEDGE ASSESSMENT AND NURSING TEAM IN THE \\ OCCURRENCE OF SEPSIS.
}

Cecília de Souza Melech 1; Maria Cristina Paganini2;

\section{RESUMO}

OBJETIVO: Avaliar o conhecimento dos profissionais de Enfermagem e Medicina na identificação das ocorrências de sepse com o objetivo de implantar ações para a redução de morbimortalidade de pacientes vitimados por essa síndrome. MÉTODOS: Trata-se de um estudo de campo com abordagem quantitativa realizado em uma unidade de urgência e emergência, em um hospital público na cidade de Curitiba-PR, no período de agosto até setembro de 2015, cuja amostra inicial era de 236 profissionais, sendo 65 médicos, 31 enfermeiros, 39 técnicos e 101 auxiliares de enfermagem. Para avaliar o conhecimento desses profissionais foi aplicado um instrumento com perguntas e análise de 5 estudos de casos sobre a identificação de sinais e sintomas de sepse. RESULTADO: 92 profissionais participaram do estudo, sendo entre eles 27 médicos, 24 enfermeiros, 10 técnicos de enfermagem e 31 auxiliares de enfermagem. A partir da análise de dados, verificou-se um conhecimento inadequado pelos profissionais, tanto no reconhecimento de sinais e sintomas relacionados a SIRS e a disfunção orgânica, como também na identificação dos quadros clínicos. Com relação a sequência de atendimento ao paciente séptico nas primeiras 6 horas, apenas 17,4\% responderam corretamente, contudo, referente ao momento correto para coleta de culturas, $81,5 \%$ do total dos profissionais responderam corretamente. CONCLUSÕES: Visto que o conhecimento desses profissionais está diretamente ligado a identificação e tratamento precoce da síndrome séptica, acredita-se que a realização dessa pesquisa possa colaborar para implantação de ações que visem diminuir o índice de morbimortalidade por essa síndrome.

Descritores: SIRS, Disfunção orgânica, Sepse, Tratamento Precoce

\section{ABSTRACT}

OBJECTIVE: To assess the knowledge of nursing and medical professionals in identifying sepsis occurrences in order to implement actions to patient morbidity and mortality reduction victimized by this syndrome. METHODS: This is a field study with a quantitative approach carried out in an emergency unit and emergency, in a public hospital in the city of Curitiba, PR, from August to September 2015, the original sample was 236 professionals, and 65 doctors, 31 nurses, 39 technicians and 101 nursing assistants. To assess the knowledge of these professionals an instrument with questions and analysis of five case studies on the identification of signs and symptoms of sepsis was applied. RESULTS: 92 professionals participated in the study, including 27 doctors, 24 nurses, 10 nursing technicians and 31 nursing assistants. From the data analysis, it was found inadequate knowledge by professionals, in recognition of the signs and symptoms related to SIRS and organ dysfunction, as well as the identification of clinical pictures. Regarding the sequence of care septic patients within 6 hours, only $17.4 \%$ answered correctly, however, regarding the right time to collect cultures, $81.5 \%$ of the professionals answered correctly. CONCLUSIONS: As the knowledge of these professionals is directly linked to early identification and treatment of sepsis syndrome, it is believed that the realization of this research may contribute to implementation of actions aimed at reducing the mortality rate from this syndrome.

Keywords: SIRS, Organic dysfunction, sepsis, Early Treatment;

\footnotetext{
1 - Acadêmica de enfermagem pela Faculdade de Ciências Biológicas e da Saúde da Universidade Tuiuti do Paraná.

2- Doutora em Enfermagem. Docente do Curso de Enfermagem da Faculdade de Ciências Biológicas e da Saúde da Universidade Tuiuti do Paraná.
}

Contato do Autor / Mail to:

Cecília de Souza Melech - cecilia.melech@gmail.com

Avenida Senador Salgado Filho, 4728; Curitiba/PR CEP 81.570-001 


\section{INTRODUÇÃO}

A sepse é definida como uma síndrome clínica constituída por uma resposta inflamatória sistêmica associada a um foco infeccioso, caracterizada por manifestações múltiplas e que pode determinar disfunção ou falência de um ou mais órgãos, podendo ser causada por bactérias, vírus, fungos ou protozoários ${ }^{1}$.

Seu diagnóstico é clínico e baseia-se pelo menos em duas alterações que constituem a Síndrome da Resposta Inflamatória Sistêmica (SIRS) que são: temperatura corporal $>38 \mathrm{C}$ ou hipotermia corporal $<36 \mathrm{C}$, taquicardia com a frequência cardíaca $>90 \mathrm{bpm}$, taquipnéia com frequência respiratória $>20 \mathrm{rpm}$ ou $\mathrm{PaCO} 2$ $<32 \mathrm{mmHg}$, leucocitose ou leucopenia $>12.000 \mathrm{cel} / \mathrm{mm} 3$ ou $<4.000 \mathrm{cel} / \mathrm{mm} 3$, ou a presença de $>10 \%$ de formas jovens (bastões) ${ }^{2}$.

Atualmente ela é considerada um problema de saúde pública e as estimativas apontam a ocorrência de aproximadamente 600 mil novos casos a cada ano no Brasil. Este aumento na ocorrência tem impacto direto nos indicadores de morbimortalidade, responsáveis pelas causas de $16,5 \%$ dos atestados de óbitos emitidos no Brasil, ou seja, em torno de 250 mil casos ${ }^{3}$.

Desta maneira, ao longo dos anos a sepse tem se tornado um desafio no tratamento de pacientes graves por estar relacionada a fatores como alta incidência, alta mortalidade e altos custos, o que têm contribuído para o direcionamento de grandes esforços com o objetivo de se entender melhor essa síndrome 4 .

Assim, este crescente interesse tem mudado o perfil de atuação de pesquisadores, profissionais, gestores de saúde e órgãos governamentais. Não mais encarada como uma complicação eventual, a sepse direciona as atenções destes profissionais no sentido de reduzir sua incidência e a taxa de mortalidade 5 .

Nesta discussão, um dos pontos importantes para a redução da mortalidade dos pacientes sépticos é através da pronta identificação dos sinais e sintomas. A prestação imediata da assistência é fundamental para um bom prognóstico, minimizando as possíveis consequências, como por exemplo, a ocorrência de falência orgânica ou até mesmo a morte do paciente6.

Tendo em vista a importância do papel desses profissionais frente ao paciente séptico, essa pesquisa tem como objetivo avaliar o conhecimento de médicos e equipe de enfermagem acerca de sepse, sepse grave e choque séptico, que atuam em uma unidade de urgência e emergência em um hospital público do município de Curitiba-PR, Brasil.

\section{MÉTODOS}

A pesquisa seguiu as normas brasileiras de pesquisa que envolve seres humanos respeitando a resolução 466/2012. Este projeto foi encaminhado para avaliação do Comitê de Ética em Pesquisa em Seres Humanos do HC - UFPR, tendo sido aprovado e registrado na Plataforma Brasil sob o número CAAE 47281015.0.0000.0096. Estas etapas foram realizadas antes do início da coleta de dados.

Trata-se de um estudo de campo de caráter exploratório com abordagem quantitativa realizado no período de agosto até setembro de 2015 , em uma unidade caracterizada por prestar assistência de urgência e emergência ao adulto, não resultante de trauma, e para pacientes classificados como críticos.

O local do estudo foi a Unidade de Urgência e Emergência, formada por serviços de Pronto Atendimento (PA), Centro de Terapia Intensiva (CTI), Centro de Terapia Semi-Intensiva (CTSI). Pertencente a um hospital público sediado no município de Curitiba-PR, considerado de grande porte e de nível terciário, destinado à assistência, ao ensino, pesquisa e extensão.

Os profissionais de saúde selecionados para participar desse estudo foram aqueles que trabalhavam nesta Unidade de Urgência e Emergência e estavam relacionados com a equipe de enfermagem ou médica.

0 tamanho da amostra inicial estimado era de 236, sendo 65 médicos, 31 enfermeiros, 39 técnicos e 101 auxiliares de enfermagem, contudo, devido à greve de funcionários no período de coleta de dados, o número de funcionários presentes estava reduzido.

Para coleta de dados utilizou-se um instrumento (em suplemento) elaborado pela própria pesquisadora, composto por questões objetivas referentes ao conhecimento sobre a síndrome séptica e cinco estudos de casos com opções fechadas para respostas à cerca da análise de ocorrências de sepse, sepse grave e choque séptico e espaço aberto para justificativa da resposta.

O instrumento foi embasado em uma busca de revisão de literatura seguindo as Diretrizes da Campanha Sobrevivendo à Sepse (2012), além de passar por um processo de avaliação feito por membros do grupo de estudo de sepse.

Para a análise dos dados foi utilizado o software IBM SPSS Statistics Base versão 12, com intuito da análise estatística. Para a criação de tabelas e gráficos utilizou-se os dados em planilhas do Excel.

\section{RESULTADOS}

Participaram desta pesquisa 92 profissionais, dos quais 27 eram médicos, 24 enfermeiros, 10 técnicos de enfermagem e 31 auxiliares de enfermagem.

Ao analisar os dados sócios demográficos verificou-se que $80,4 \%$ eram do sexo feminino e $19,6 \%$ do sexo masculino, com uma prevalência de idade entre 30 a 40 anos. 
Como pode ser observado no gráfico 1 , quanto ao reconhecimento dos sinais e sintomas diferenciando-os entre SIRS e disfunção orgânica, verificou-se que os sinais com mais de $50 \%$ de acerto entre todas as categorias foram temperatura $>38^{\circ} \mathrm{C} 87 \%$, frequência respiratória > $20 \mathrm{rpm}$ 75\%, frequência cardíaca > 90 bpm 67,4\% leucocitose $>12$ mil $65,2 \%$ e alteração do estado mental $51,1 \%$, enquanto os outros sinais e sintomas atingiram porcentagens inferiores a $50 \%$.

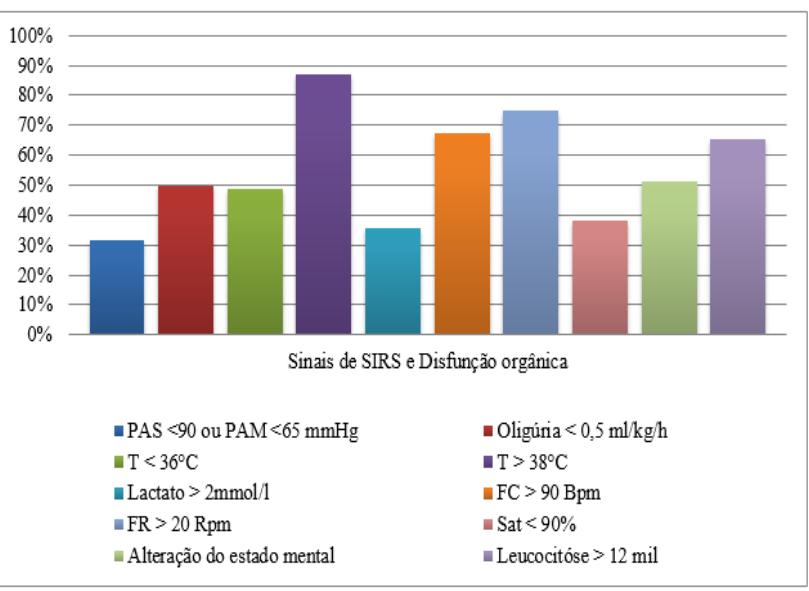

Gráfico 1: Percentual de acertos dos profissionais na identificação de sinais de SIRS e disfunção orgânica.

Com relação à sequência correta de atendimento ao paciente com sepse grave nas primeiras 6 horas, apenas $17,4 \%$ do total dos profissionais responderam a sequência correta: Coleta de lactato, coleta de culturas, antibiótico, reposição volêmica, vasopressor, obtenção de cateter venoso central e manter $\mathrm{SatO}^{2}$, sendo $18,5 \%$ médicos, 29,2\% enfermeiros, $10 \%$ técnico em enfermagem e $9,7 \%$ auxiliares de enfermagem, enquanto $82,6 \%$ marcaram alternativas erradas.

Referente ao momento correto para realizar a coleta de culturas $81,5 \%$ dos participantes assinalaram a alternativa correta e $18,4 \%$ marcaram as alternativas erradas ou não informaram.

Quanto ao tempo ideal para iniciar o tratamento com o antibiótico em pacientes diagnosticados com sepse $63 \%$ do total dos profissionais responderam corretamente: Em até uma hora, e $37 \%$ marcaram respostas erradas ou não souberam responder, sendo que os médicos foram os que mais acertaram ficando com $92,6 \%$.

Em relação às análises dos estudos de casos clínicos, o primeiro caso referente ao paciente com diagnóstico de choque séptico, apresentou 40,2\% de acerto e $44,6 \%$ erraram as respostas, enquanto $15,2 \%$ responderam que não sabiam identificar o quadro clínico. Com relação às justificativas após analisadas, percebeu-se que $42,4 \%$ não responderam e apenas 35,8\% responderam corretamente relacionando o quadro a choque séptico devido à hipotensão não responsiva a reposição vigorosa de volume.
No primeiro caso de sepse grave, os resultados das respostas dos participantes indicaram que 29,3\% acertaram confirmando diagnóstico de sepse grave, 18,5\% marcaram que não sabiam responder e 52,3\% marcaram respostas erradas. Com relação à justificativa sobre a disfunção orgânica, $50 \%$ não informaram e 28,2\% justificaram corretamente, sendo desses 26 profissionais $85,2 \%$ médicos e $12,6 \%$ enfermeiros.

No segundo caso de sepse grave, as respostas demonstraram que $43,5 \%$ acertaram, do restante $16,3 \%$ marcaram que não sabiam responder e 40,2\% erraram a resposta. Quanto às justificativas, 53,3\% não informaram e $19,6 \%$ justificaram corretamente citando as disfunções orgânicas como critério de avaliação.

No estudo de caso clínico que indicava sinais de sepse, $21,7 \%$ acertaram o diagnóstico de sepse, e 22,8\% responderam que não sabiam identificar o quadro clínico do paciente. Analisando as justificativas apenas 19,6\% acertaram, justificando apenas critérios de SIRS com provável foco infeccioso, enquanto $58,7 \%$ não responderam.

Já no caso clínico que apresentava paciente sem diagnóstico sugestivo para sepse. Os resultados das respostas indicaram que apenas 32,6\% acertaram, 16,3\% disseram que não sabiam informar qual era a resposta correta e $55,5 \%$ não souberam responder corretamente. Com relação às justificativas, apenas $7,6 \%$ acertaram afirmando que o quadro não condiz com sepse, enquanto $53,3 \%$ não souberam responder, como apresenta os gráficos 2 e 3.

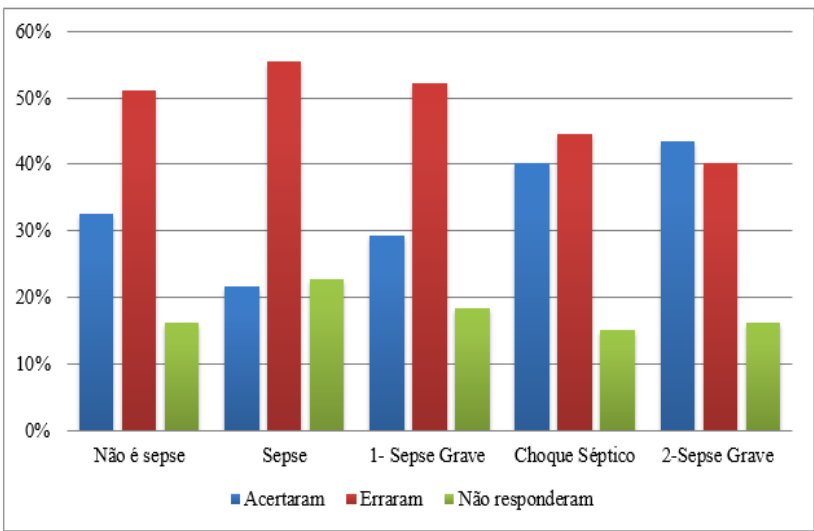

Gráfico 2: Percentual de acertos dos profissionais em relação aos estudos de casos clínicos.

\section{DISCUSSÃO}

Ao analisar os resultados, verificou-se a predominância de mulheres entre os profissionais, com $80,4 \%$ do total dos profissionais, este achado reafirma outros estudos que apontam a característica do perfil dos profissionais da saúde que trabalham em hospitais no Brasil como predominantemente do sexo feminino ${ }^{7}$. 
Em relação ao reconhecimento de sinais e sintomas relacionados a SIRS e a disfunção orgânica por parte dos profissionais identificou-se certa dificuldade em

Gráfico 3: Percentual de profissionais que responderam as justificativas.

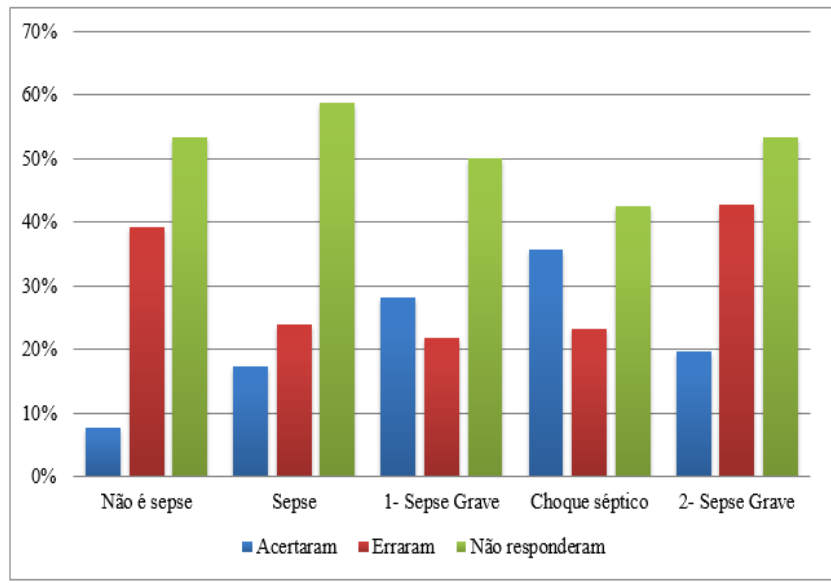

identificar quais sinais são relacionados à disfunção orgânica. Apenas cinco sinais relacionados à SIRS obtiveram mais de $50 \%$ de acerto, enquanto sinais de disfunção orgânica como hipotensão com pressão arterial sistólica < $90 \mathrm{mmHg}$ e pressão média arterial < $65 \mathrm{mmHg}$, lactato $>2 \mathrm{mmol} / \mathrm{I}$ e Saturação < $90 \%$ os quais são imprescindíveis na detecção de evolução do quadro clínico, ficaram a baixo de $40 \%$.

Com relação aos estudos de casos clínicos, dos cinco casos apenas o quadro clínico do paciente sugestivo para sepse grave, onde o fator principal marcador de disfunção orgânica era oligúria teve um percentual de acerto maior que o de erro, nos outros casos clínicos sugestivos para sepse, sepse grave, choque séptico e não sugestivo para sepse, tiveram percentual de erro maior que o de acerto

Ao analisar as justificativas por se tratarem de questões abertas, nota-se que a maioria dos participantes não respondeu. Cabe ressaltar que nos únicos casos em que as justificativas estão corretas são nos casos de choque séptico e no primeiro caso de sepse grave.

Neste sentido, quando comparamos o resultado do conhecimento dos profissionais em relação ao reconhecimento dos sinais de SIRS e disfunção orgânica com o resultado da análise dos estudos de casos clínicos e suas justificativas, nota-se que a falta desse conhecimento está diretamente relacionada com o baixo percentual de acerto dos estudos de casos clínicos e suas justificativas.

Segundo Almeida8 et al (2013) o reconhecimento das manifestações clínicas associadas ao quadro de sepse é fundamental para a classificação correta do paciente.

Cabe ressaltar que a demora no diagnóstico pode levar a uma evolução do quadro clínico do paciente, de sepse para sepse grave ou até mesmo para choque séptico. Essa evolução no quadro leva a um aumento do tempo de internação e ao aumento de comorbidades como disfunções de órgãos, sendo estes fatores diretamente associados ao maior risco de morte 8 .

Estudo realizado em Unidade de Terapia Intensiva mista de novembro de 2003 a fevereiro de 2004 , no Brasil, ressalta que o risco de mortalidade aumenta em 8,7 vezes para os pacientes que são diagnosticados após 48 horas com disfunção orgânica. Ou seja, quanto mais tempo demorar a diagnosticar a disfunção orgânica, maior a chance de piora no quadro clínico do paciente, podendo até levá-lo a morte ${ }^{10}$. Dessa maneira, percebemos a importância das equipes médicas e de enfermagem no reconhecimento dos sinais de disfunção orgânica. Torna-se assim, indispensável que a equipe responsável por avaliar esses pacientes saiba reconhecer e compreender todos os sinais e sintomas do quadro. Gerando a partir desse reconhecimento medidas de tratamento com o menor tempo possível, reduzindo o risco de complicações ocasionadas por diagnóstico tardio11.

Quanto à sequência correta de atendimento ao paciente com sepse grave nas primeiras 6 horas, verificouse o baixo percentual de acerto entre os participantes, sendo a sequência correta: Coleta de lactato, coleta de cultura, antibiótico, reposição volêmica, vasopressor, obtenção de cateter central e manter SatO².

A sequência de atendimento ao paciente com sepse grave nas primeiras 6 horas foi preconizada pelas Diretrizes Internacionais para Tratamento de Sepse Grave e Choque Séptico de 2012, tendo como justificativa, a análise de estudos já referenciados, comprovando que o atendimento correto no período inicial de 6 horas está associado a uma redução de $15,9 \%$ na taxa de mortalidade 6 .

Segundo Peninck e Machado12 (2012) as seis primeiras horas após o diagnóstico constituem a janela de oportunidade no tratamento da sepse, e a correta terapia aplicada dentro dessa fase inicial é capaz de reduzir a mortalidade da sepse grave e choque séptico em aproximadamente $16 \%$.

Referente ao momento correto para coleta de culturas, $81,5 \%$ do total dos profissionais respondeu corretamente: No momento em que foi dado diagnóstico, antes do início do antibiótico.

Conforme Manual de Abordagem Inicial da Sepse Grave e Choque Séptico13 (2012) a coleta deve ser realizada antes da administração do antibiótico, pois é necessário para confirmar a infecção e permitir o reconhecimento dos patógenos responsáveis, além de adequar a antibióticoterapia de acordo com o resultado de sensibilidade do antibiograma.

De acordo com o Ministério da Saúde (2009)14 nos casos de sepse as coletas de cultura constituem o principal meio de diagnostico etiológico disponível na prática clínica, a identificação do agente etiológico causador da sepse é de extrema importância, pois há evidências de que pacientes com sepse que receberam antibioticoterapia adequada ao perfil de sensibilidade do agente infeccioso tiveram menor letalidade. 
Referente ao tempo de início de administração do antibiótico, 63\% responderam corretamente: Em até 1 hora, após o diagnóstico, sendo que o tempo de início da administração de antibióticos é imprescindível no tratamento adequado ao paciente séptico, assim como afirma Salomão $(2011)^{15}$, que a antibioticoterapia deve ser iniciada logo após o diagnóstico de sepse grave ou choque séptico.

Estudos apontam que a sobrevida dos pacientes com choque séptico se reduz a cada hora de atraso do antibiótico, sendo que dentro das primeiras 6 horas após o início da hipotensão, cada hora de atraso na administração do antibiótico está associada a uma redução na sobrevida de $7,6 \% 16$.

Dessa maneira, percebe-se a importância da equipe multiprofissional focada na identificação e tratamento de sinais e sintomas indicativos para alguma alteração, impedindo assim a evolução do quadro séptico diminuindo o de morte associado à sepse grave e ao choque séptico11.

\section{CONCLUSÃO}

Conclui-se que o conhecimento acerca de sepse, sepse grave e choque séptico por parte dos profissionais de enfermagem e medicina que atuam na unidade de urgência e emergência estudada ainda é restrito e precisa ser ampliado.

Além disso, visto que este conhecimento está diretamente ligado à identificação precoce e ao tratamento da síndrome séptica, acredita-se que a realização dessa pesquisa e seus resultados possam colaborar para implantação de ações que visem garantir maior habilidade e competência a esses profissionais sobre este tema.

Tendo em vista que por se tratar de um quadro clinico em que o diagnóstico e as ações de intervenção e tratamento ainda estão intimamente relacionados à pessoa do médico, faz-se necessário novos estudos que identifiquem de maneira mais completa como envolver os profissionais de enfermagem e demonstrar o importante papel que exercem na prevenção da instalação do quadro mais grave de sepse.

Também é importante ressaltar que o desafio do reconhecimento da sepse tornou-se ainda mais significativo diante da recente publicação das novas definições de sepse (SEPSE-3), publicadas em fevereiro de 2016 pelo JAMA (The Journal of American Medicine Association), que suprimem 0 uso de SIRS para o diagnóstico desta síndrome, propondo o uso do qSOFA (quick SEQUENTIAL ORGAN FAILURE ASSESSMENT SCORE) como definidor de sepse, além da eliminação do termo sepse grave, passando a ser utilizado apenas sepse e choque séptico.

\section{CONFLITOS DE INTERESSE}

Não houve conflitos de interesse.

\section{REFERÊNCIAS}

1. CARVAlHo P; TROTTA EA. Avanços Nos Diagnósticos E Tratamentos Da Sepse. Jornal de Pediatria. Vol. 79, Supl.2, 2003

2. NETO, JMR., et al. Assistência de Enfermagem a Pacientes Sépticos Em Uma Unidade De Terapia Intensiva Adulto. Facene/Faceme - 9(2) 2011.

3. BRASIL. Conselho Regional De Medicina. Estudos da Sepse: Um problema de saúde pública. p.90, Brasília, 2015.

4. INSTITUTO LATINO AMERICANO DE SEPSE. Campanha De Sobrevivência à Sepse, Atendimento ao paciente com sepse grave/choque séptico. Disponivel em $<$ www.llas.org.br $>;<$ http://www.sepsisnet.org/pg $>;<\underline{h}$ ttp://www.ilas.org.br/upfiles/aula03/index1.swf $>$ Ace sso em 30 de outubro de 2015.

5. SILVA, E. Sepse, um problema do tamanho do Brasil. Revista Brasileira Terapia Intensiva Volume 18 Número 1 - Janeiro/Março 2006.

6. DELLINGER, RF et al. Campanha de Sobrevivência à Sepse: Diretrizes internacionais para tratamento de sepse grave e choque séptico: 2012. Critical Care Medicine N.2 Vol. 41, Fevereiro, 2013

7. FRONZA, FC; TEIXEIRA, LR. Perfil dos Profissionais de Saúde que Trabalham em Hospitais: Relação entre Sintomas Musculoesqueléticos e Qualidade de Vida. Revista Brasileira de Ciencias da Saúde, ano , $n^{\circ} 24$, abril/junho 2010.

8. ALMEIDA, AP et al.Conhecimento do Profissional Enfermeiro a Respeito da Sepse. Brazilian Journal of Surgery and Clinical Research- BJSCR Vol.4, n.4,PP.05-10 Set-Nov 2013

9. KOURY, JC; LACERDA,HR; NETO, AJB. Fatores de Risco Associados a Mortalidade em Pacientes com Sepse em Unidade de Terapia Intensiva de Hospital Privado de Pernambuco. Revista Brasileira de Terapia Intensiva. São Paulo, Vol. 19 No 1, Janeiro - Marco, 2007

10. FREITAS, FGR et al. The impacto of Duraction of Organ Dysfunction oh the Outcome of Patients With Severe Sepsis and Septic Shock. Clinics 64:483-8, 2008

11. WESTHENPHEL, GA et AL. Estratégia de detecção precoce e redução de mortalidade na sepse grave. Revista Brasileira de Terapia Intensiva. 2009; 21(2):113 123.

12. PENINK, PP; MACHADO, RC. Aplicação Do Algoritmo Da Sepse Por Enfermeiros Da Unidade De Terapia Intensiva. Revista Rene. 2012; 13(1): 187-99.

13. BRASIL. Ministério da Saúde, Sociedade Beneficente Israelita Brasileira Hospital Albert Einstein, Instituto Latino Americano de Sepse. Manual de abordagem 
inicial da sepse grave e choque séptico, São PauloSP, $1^{\mathrm{a}}$ edição - 2012.

14. BRASIL. Ministério Da Saúde Do Brasil. Primeiras Diretrizes Clínicas na Saúde Suplementar - versão preliminar. $1^{\circ}$ Edição, Rio de Janeiro 2009.

15. SALOMÃO, R et al. Diretrizes para tratamento da sepse grave/choque séptico: abordagem do agente infeccioso - controle do foco infeccioso e tratamento antimicrobiano. Revista Brasileira de Terapia Intensiva. 2011; 23(2):145-157.

16. ANAND KUMAR, MD et al. Duraction of Hypotesion Before Initiation of Effective Antimicrobial Therapy is the Critical Determinant of Survival in Human Septic Shock. Critical Care Med, vol. 34, N 6, 2006 